\title{
LUGAR DE IDENTIDADE: TEMPO E ESPAÇO NAS CONSTRUÇÕES IDENTITÁRIAS
}

\author{
Raphaely Pereira Fernandes ${ }^{1}$ \\ Claudineia Vicente de Almeida ${ }^{2}$ \\ Elloyna Duarte da Silva ${ }^{3}$ \\ Gabriela da Silva Pedroso 4 \\ Laura Maria Barcha Giroldo 5 \\ Maria Cristina Marcelino Bento ${ }^{6}$ \\ Luiz Antonio Feliciano ${ }^{7}$
}

Resumo: A pesquisa destaca os conceitos de lugar, na busca de identidades, que propõe reconstruir a tessitura da história de uma instituição escolar com mais de 60 anos de existência, em uma cidade no interior do Estado de São Paulo. O objetivo principal dessa proposta se consolida na problematização de uma identidade que permeie a vivência em um espaço escolar, mas que, também, extrapole os muros da Instituição. Essa é uma maneira de entender a relação do sujeito com os espaços de sua vivência e a importância do lugar na sua construção como pessoa. Por conseguinte, o desenvolvimento do trabalho possibilita discutir o papel da fotografia como registro das narrativas e como suporte visual da memória. $O$ método utilizado será o perfil histórico-sociológico e a coleta dos dados se dará por meio dos relatos orais de alunos do ensino médio, do Instituto Santa Teresa, de graduandos, professores e funcionários, do UniFatea, e de membros da comunidade, que têm uma participação efetiva na cotidianidade da Instituição e da sua história. Narrativas que, por hipótese, corroboram para o sentimento de pertencimento a um grupo e um lugar.

Palavras-chave: Identidade; Experiência; Narrativa; Lugar; Temporalidade.

\footnotetext{
1 Publicidade e Propaganda/UniFatea, Brasil. E-mail: raphaelypfernandes@gmail.com.

2 Pedagogia/UniFatea, Brasil. E-mail: claudineia.vicente@yahoo.com.br.

3 Jornalismo/UniFatea, País. E-mail: elloynabarbosa@gmail.com.

${ }^{4}$ Rádio, TV e Internet/UniFatea, Brasil. E-mail: gs-pedroso@bol.com.br.

5 Jornalismo/UniFatea, Brasil. E-mail: laurabarcha@gmail.com.

6 Pedagogia/UniFatea, Brasil. E-mail: criscabento@gmail.com.

7 Publicidade e Propaganda/UniFatea, Brasil. E-mail: luizliu@yahoo.com.br.
} 